Journal of Advanced Research in Fluid Mechanics and Thermal Sciences

Journal homepage: www.akademiabaru.com/arfmts.html ISSN: 2289-7879

\title{
Experimental Investigation in Improving Thermal Performance of Passive Heat Removal System using Mist Assisted Evaporative Cooling
}

\author{
Akram Hamzah Abed ${ }^{1,2,}{ }^{*}$, Sergey E. Shcheklein ${ }^{1}$, Valery M. Pakhaluev ${ }^{1}$ \\ 1 Department of Nuclear Power Plants and Renewable Energy Sources, Ural Power Engineering Institute, Ural Federal University, 19 Mira St., \\ Yekaterinburg 620002, Russia \\ Department of Electromechanical Engineering, University of Technology, Baghdad, Iraq
}

\section{ARTICLE INFO}

\section{Article history:}

Received 15 December 2019

Received in revised form 29 January 2020

Accepted 29 January 2020

Available online 7 April 2020

\section{Keywords:}

PHRS; water mist; thermal performance factor

\section{ABSTRACT}

Demands to increase the safety and reliability of the modern nuclear reactors are constantly being made. Typical demands include the increases the thermal performance of the passive heat removal system PHRS by removing larger amounts of energy and applied a technique that leads to reduce size and weight of the PHRS unit. This article presents an experimental investigation on the natural convection based on alternative cooling approach using water mist. The results obtained from the related experimental work indicated that the heat transfer rate was enhanced over that for the airflow as a result of water mist evaporation on the surface of the tubes. The Nu number increases by about $148 \%, 144 \%$, and $128 \%$ respectively for all tube rows compared with airflow. The experimental results of the thermal performance factor values obtained by suspended water mist were correlated as functions of Rayleigh number (Ra) and Weber number (We). Subsequently, the predicted thermal performance factor from the correlation was plotted to compare with the experimental data. It was found that the thermal performance factor was within $\pm 11 \%$.

Copyright @ 2020 PENERBIT AKADEMIA BARU - All rights reserved

\section{Introduction}

After Fukushima Daiichi disaster, the passive heat removal system PHRS become very important in nuclear power plants NPPs to improve the safety and reliability of the nuclear reactors. The PHRS is aimed to protect the primary circuit by removing the decay heat from the reactor core in an emergency shutdown or blackout conditions [1]. In the Water-Water Energetic Reactor-WWER or VVER design, the PHRS system comprises of four independent coolant circuits, each one involves heat exchange modules, air gates, steam pipelines and air ducts to supply the ambient air and bleed the heated air as shown in Figure 1. In normal operation conditions of NPPs, the air gates are closed and

\footnotetext{
* Corresponding author.

E-mail address: akraaam82@yahoo.com (Akram Hamzah Abed)
} 
PHRS circuits are in a standby mode. During NPPs blackout condition, the steam that is generated in the steam generator (SG) due to the released heat from the reactor core condenses and subtracts its heat to the ambient air by the PHRS system [2]. The heat dissipation from the outer surface of the PHRS to surrounding air based on natural forces, such as gravity and buoyancy induced-natural circulation without any external power. Unfavorable several ambient factors such as air temperature, solar radiation, crosswind, rain, and snow can indeed be affected on the PHRS operation mechanism [3]. In hot-arid or subtropical climates regions such as Iran, Bangladesh, Egypt, Saudi Arabia, etc, with high ambient temperature (Up to $45^{\circ} \mathrm{C}$ in summer) have an additional challenge when designing the PHRS modules. A coupled thermal and hydraulic geometrical calculation of PHRS for several boundary conditions and geometrical combinations with reference to station blackout scenario was performed by [4]. They highlighted that the rejectable heat amount of PHRS decreased by 2-3 kW with 1-kelvin increases in ambient temperature. They also found that the PHRS performance significantly depends on the system elevation and the rejected heat does not influenced by the length of pipe in horizontal segments. The removal heat from PHRS is essential to increase the safety and reliability of the nuclear reactors and avoid the accidents after reactor shutdown. Therefore, enhancing the PHRS system is of great importance. So, increasing the heat transfer area can be used to improve the heat transfer rate between the PHRS surface and the surrounding medium in the form of grooves, ribs, and extended surfaces attached to surfaces [5-6]. Thermal-hydraulic computations of PHRS have been derived in the case of the finned tube structure by [7]. The effects of several parameters such as total fin number, fin radius, and fin thickness were examined. The computation results gave an idea about the geometrical specifications and capacity of PHRS of VVERs. The essential fin parameter was the fin radius and required tube length decreases as the fin radius increases. Another important parameter is the fin thickness. They pointed out that fin thickness must be thin to avoid the pressure drop impact that ultimately reduces the PHRS heat transfer performance. In another studies [8-12], the heat transfer and hydraulic resistance of helical fins on the internal surface of tubes have been investigated experimentally and numerically under a wide range of operating parameters. All these heat transfer enhancement methods have provided a great augmentation in PHRS performance, but the ambient temperature still plays an important role especially in the hot summer days. Accordingly, advanced cooling technologies are needed to meet the operating conditions and developing more compact and efficient PHRS that are still working at high ambient air temperature.

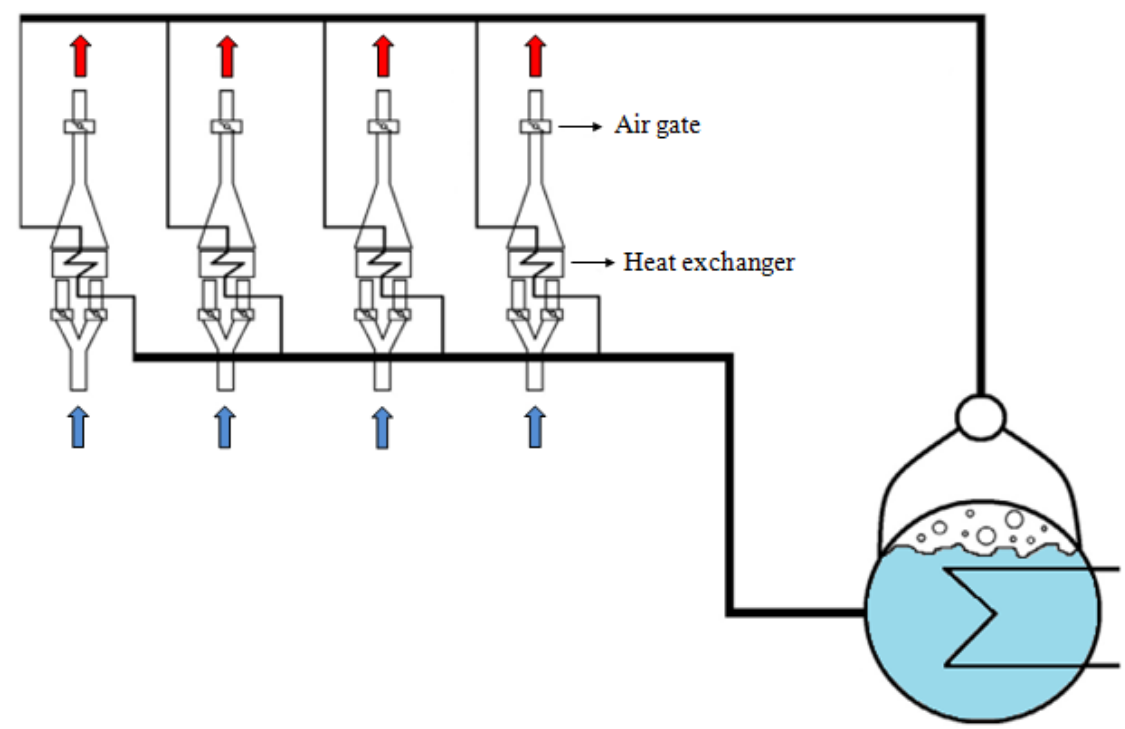

Fig. 1. The simplest diagram of PHRS 
In this study, a water droplet cooling technique or (water mist cooling technique) were developed by suspending fine water droplets into the working fluid. The water mist techniques, not a new cooling concept and has been successfully applied in many industrial applications [13-18]. Utilizing water mist as a cooling medium provides excellent cooling approaches to dissipate heat even larger than the single-phase air cooling approaches.

So, our objective in this study is to experimentally investigating the natural convection heat transfer enhancement of a staggered horizontal tube bundle using an alternative cooling approach by water mist, which takes advantage of the substantial latent heat of water evaporation. In this regard, the influence of Ra number and the water mist rate on heat transfer characteristics were verified.

\section{Experimental Apparatus}

An experimental apparatus has been constructed to simulate the PHRS and investigate the water mist effect on natural convection heat transfer characteristics. The experimental apparatus is an open-loop system and it mainly consists of an isolated channel having a rectangle cross-section of $117 \times 55 \mathrm{~mm}$ is set vertically, tube bundle, heating element, data acquisition system and water mist system. A schematic layout and photographic view of the experimental apparatus is shown in Figures 2 and 3 . The staggered tube bundle consists of three rows of tubes of $14 \mathrm{~mm}$ outside diameter and $55 \mathrm{~mm}$ length. The test tube was constructed from a copper rod and each one was heated internally by using A.C. cartridge heater with $100 \mathrm{~W}$ maximum power.

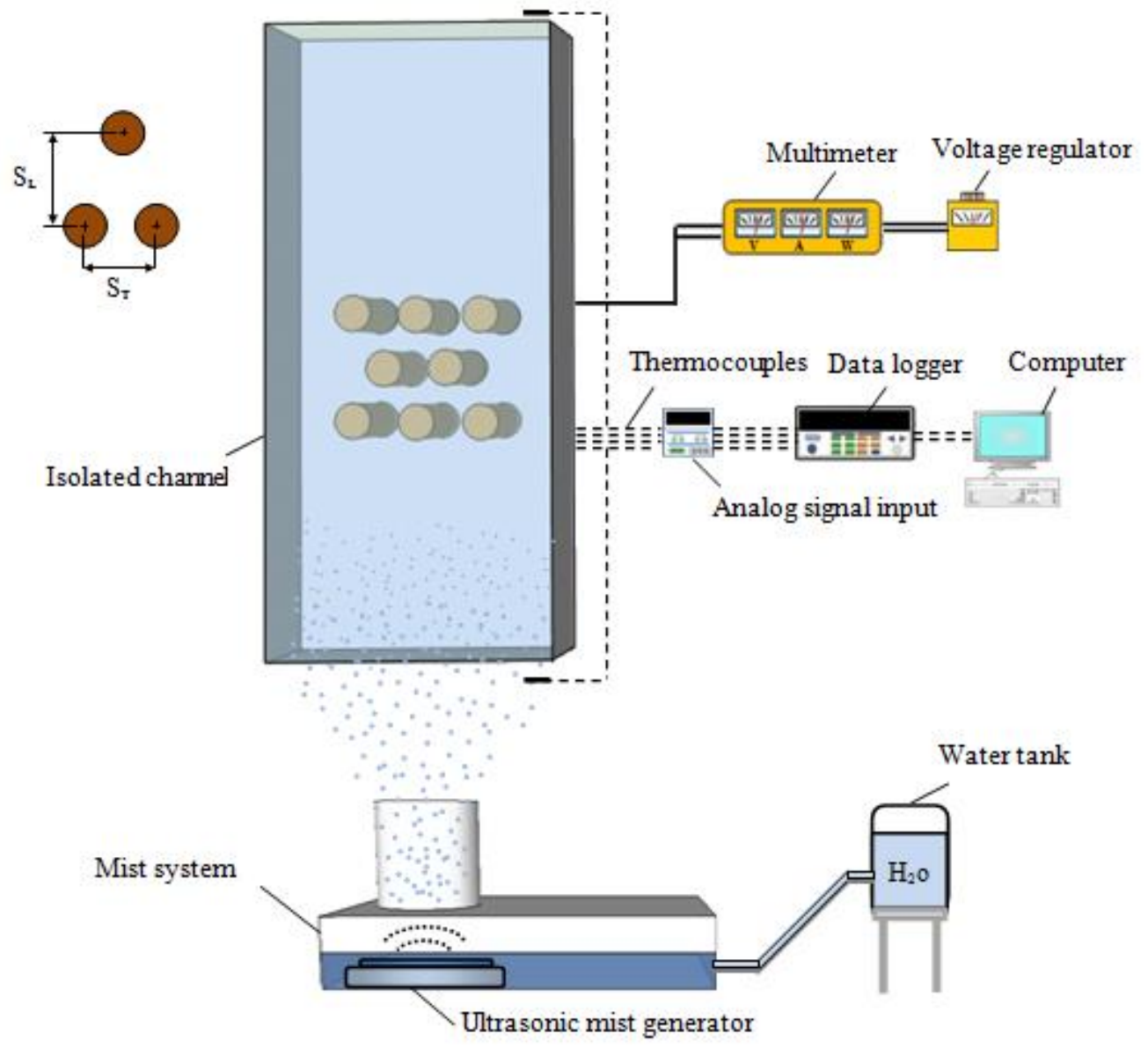

Fig. 2. Schematic diagram of the experimental apparatus 


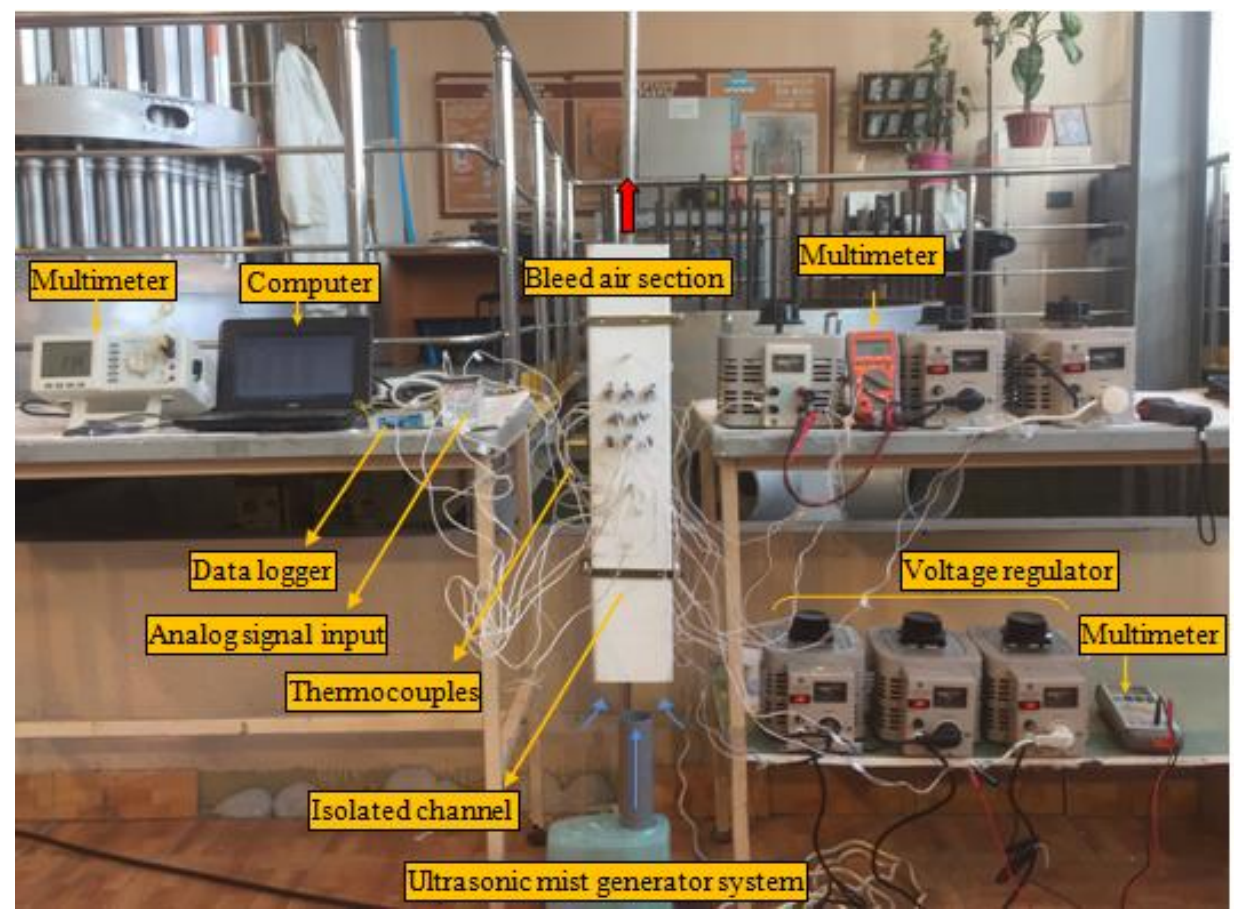

Fig. 3. Photographic views of the experimental apparatus

A variac voltage regulator was used to control the heater input power and achieve the heat flux required. To boost the thermal conductance between the tubes and the A.C cartridge heater, high thermal conductivity material was applied. The pitches of the tube bundle were chosen to be 2.14 and 2.85 of tube diameter in the transverse and longitudinal directions. To measure the surface temperature, Eight k-type thermocouples are implanted on the backside of the tubes with $180^{\circ}$ from the forward stagnation point. In addition, the inlet and outlet air temperatures were also measured. All thermocouples are connected to a data acquisition system that consists of a signal input module with a data logging system. Water mists with micro-sized $(2-10 \mu \mathrm{m})$ were suspending in the air by an ultrasonic mist generator comprises of a piezoelectric atomizing transducer that generates a highfrequency oscillation of $1.7 \mathrm{MHz}$, and sprays the water away from the water surface to produce a natural floating water mist without any heating or mechanical agent. Due to the low energy needed and a very quiet operation condition compared to other mist and evaporative generators, this type of mist generator was selected.

\section{Methodology}

\subsection{Data Processing and Method of Calculation}

This study examined experimentally the influence of water mist rate on the natural heat transfer enhancement. In view of this, fine water droplets were suspended in the inlet air and the air/water mist was obtained as a working medium. Based on the capillary wave theory, the increases of the ultrasonic wave's frequency lead to decreases the capillary wavelength; thus, the finer mist is generated at higher frequencies. Lang [19] proposed an equation to obtain the count median diameter (CMD) of the generated water mist depends on the ultrasonic frequency as Eq. (1)

$$
d_{p}=0.34\left(\frac{8 \pi \sigma}{\rho f^{2}}\right)^{\frac{1}{3}}
$$


where $\sigma$ - the surface tension coefficient, and $f$ - the working frequency of the ultrasonic mist generator. When analyzing the interaction between the water mist flow and heated surface, it is usually considered an equation in the form of total heat carried by the mixture and water mist evaporation rate [20].

$q=\alpha_{k} \cdot S \cdot\left(T_{s}-T_{\infty}\right)+r \cdot n \cdot \beta / \alpha_{k} \cdot\left(x_{s}-x_{s a t}\right)$

where $\alpha_{k}$ - the water mist heat transfer coefficient, $S$ - surface area, $T_{s}-T_{\infty}$-surface temperature and fluid temperature, $r$ - the latent heat of evaporation, $\beta$ - mass transfer coefficient, $X_{s}-X_{\text {sat.-moisture }}$ content of saturated air by temperature $\mathrm{Ts}-\mathrm{T}_{\infty}$. According to Lewis number, Eq. (2) can be written as

$q=\alpha_{k} \cdot S \cdot\left(T_{S}-T_{\infty}\right)+\frac{r}{c_{p}} \cdot n \cdot\left(x_{s}-x_{s a t}\right)$

With a high water mist rate, the water mist can partially settle on the tube surface without evaporation in the boundary layer. Taking into account the heating of the water mist settling in the form of separate droplets or a water film to the surface temperature, the resulting heat flux from the cooled surface can be defined as

$q=\alpha_{k}\left(T_{s}-T_{\infty}\right)+j\left[r+C_{p, w}\left(T_{s}-T_{\infty}\right)\right] \cdot n$

The corresponding heat transfer coefficient can be defined as

$\alpha=\frac{q}{\left(T_{S}-T_{\infty}\right)}=\alpha_{k}+\alpha_{r}\left[1+\frac{C_{p, w}\left(T_{S}-T_{\infty}\right)}{r}\right] \cdot n$

$\alpha_{r}=\frac{j \cdot r}{\left(T_{s}-T_{\infty}\right)}$

Herein, $j-$ is the water mist rate, calculated as follow

$j=\mathrm{G} / \mathrm{S}_{\mathrm{ch}}$

where $G$ - mass of water mist per unit time, $S$-channel area. The relative mass of water mist reaching to the surface of the tubes can be estimated as

$n_{i}=\left[1-\left(\frac{\alpha_{j}}{\alpha_{k}}\right)^{-1}\right] \cdot \frac{q}{0.5 \cdot j r\left[1+\frac{C_{p}\left(T_{S}-T_{\infty}\right)}{j r}\right]}$

The Rayleigh number ( $\mathrm{Ra}$ ) can be determined as follow

$R a=G r \cdot P r$

$R a=\frac{\Delta T \cdot \rho^{2} \cdot g \cdot \beta \cdot d_{s p}^{3}}{\mu^{2}} \cdot \frac{\mu \cdot c_{p}}{k} ; \beta=-\frac{1}{\rho_{\infty}}\left(\frac{\partial \rho}{\partial T}\right)_{p}$

The Nusselt number is defined as Eq. (11) 
$N u=\frac{\alpha d_{t u b}}{k}$

The Weber number is defined as Eq. (12)

$W e=\frac{1}{N} \sum_{i}^{N} \frac{j^{2} d_{p}}{2 \rho_{w} \sigma}$

In general, the following procedure was followed for carrying out this study: firstly the A.C. cartridge heater was switched on and the input power was adjusted to achieve the heat flux required. Then, the experimental apparatus was left for 120-150 minutes to achieving a steady-state condition and every $10 \mathrm{~min}$, the thermocouple reading was recorded by means of digital thermometer. Finally, after reaching the steady-state condition, the tubes temperature, inlet and outlet fluid temperatures are measured. In the same conditions, the tube bundle was exposed to air/water mist and the temperature of the tubes were measured every 5 minutes until the thermocouples reading remain constant, a final reading was recorded. For next heat flux and the same water mist rate, the heater input power could be increased to cover another run. A subsequent run for other water mist rate was performed in the same previous procedure.

\subsection{Error Analysis and Uncertainty}

According to the Moffat and Kline [21,22], the uncertainty analyses of the experimental results are performed by computing the error associated with the measured variables and estimating its effect on the final uncertainty value. The maximum uncertainty in the parameters $\mathrm{Nu}, \mathrm{Ra}$ are $5 \%$ and $5.42 \%$ respectively.

$$
\begin{aligned}
& \left(\frac{\Delta N u}{N u}\right)^{2}=\frac{1}{N u}\left[\left(\frac{\partial}{\partial \alpha}(N u) \Delta \alpha\right)^{2}+\left(\frac{\partial}{\partial d_{s}}(N u) \Delta d_{s}\right)^{2}+\left(\frac{\partial}{\partial k_{s}}(N u) \Delta k_{s}\right)^{2}\right] \\
& \left(\frac{\Delta R a}{R a}\right)^{2}=\frac{1}{R a}\left[\left(\frac{\partial}{\partial \Delta t}(R a) \Delta t\right)^{2}+\left(\frac{\partial}{\partial \rho}(R a) \Delta \rho\right)^{2}+\left(\frac{\partial}{\partial \mu}(R a) \Delta \mu\right)^{2}+\left(\frac{\partial}{\partial d}(R a) \Delta d\right)^{2}\right]
\end{aligned}
$$

\section{Results and Discussion}

An experimental investigation on natural convection from a staggered arranged tube bundle has been conducted in air as well as water mist. Initially, the tube bundle is at a uniform heat flux and the fluid is in steady-state motion due to natural convection. The effect of water mass rate on the heat transfer enhancement has been investigated for $\left(\mathrm{j}=20.48-97.85 \mathrm{~kg} \mathrm{~m}^{-2} \mathrm{hr}^{-1}\right)$ while the Rayleigh number varies from $\left(\mathrm{Ra}=18 \cdot 10^{2} \sim 12 \cdot 10^{3}\right)$ to cover a wide range of the flow. Close to each tube, there is a thin boundary layer in which the fluid temperature changes from the maximum temperature at the surface of the tube (Ts) to the fluid temperature $\left(T_{\infty}\right)$. The heat transfer rate between the tube and the fluid is defined by the steady-state natural convection and the physical properties of the fluid are assumed to be constant. Figure 4 depicts the Nusselt number averaged for each row of tubes bundle over natural airflow. The average Nusselt number significantly increases as the Ra number increases as expected. From this figure, it is observed that the Nusselt number of the first row is the highest among the second and third rows for all range of Ra number. This can be elucidated that the flow first touches the first row, and then the second and third rows in the upper direction, the cooling process of second and third rows can be achieved by the hot air bleed from the first one. 


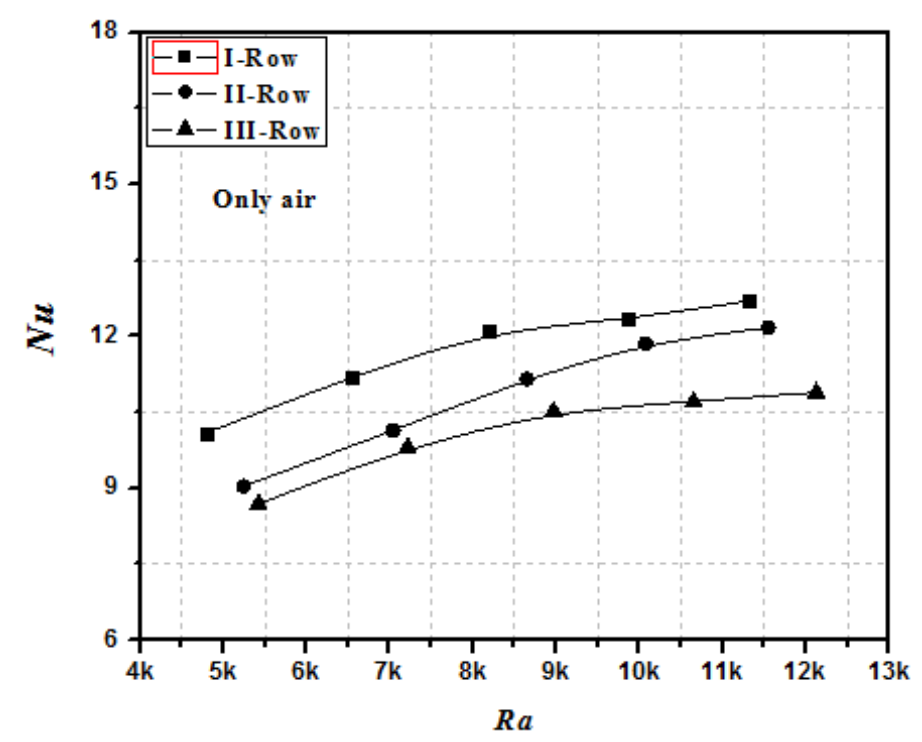

Fig. 4. Variations of Nusselt number with Rayleigh number for various tubes row

When the tube bundle exposed to water mist flow, the effect of water mist evaporation on/near the surface of the tubes appeared and can be observed that the heat transfer rate in the terms of $\mathrm{Nu}$ number increases as water mist rate increases for constant heat flux as shown in Figure 5. These indicated that the suspended water mist is an effective working fluid for thermal control and heat dissipation of heated surfaces. Also, it can be observed that the Nu number increases to a maximum value for all rows under a range of water mass rate and then decreases on increasing Ra number. These could be associated with the increase of the temperature difference due to water film formed on the large part of the tube's surface and posing an increased thermal resistance.

Therefore, the maximum Nu number may occur at a certain $\Delta T$. The Nu number increases by about $148 \%, 144 \%$, and $128 \%$ respectively under $\left(j=97.85 \mathrm{~kg} \mathrm{~m}^{-2} \mathrm{hr}^{-1}\right)$ for all tube rows compared with single-phase airflow. The heat transfer enhancement mechanism by water mist may include three leading physical process: water mist evaporation on the tubes surface (wet region), convective heat transfer (dry region), and reduce the air temperature from dry bulb to wet bulb temperature. The obtained experimental results provided a possibility to estimate the relative mass of water mist reaching to the surface of the tubes before they evaporate in the boundary layer.

Figures 6 depicted the ratio of the effective wet area to the total heat transfer area of the tube as a function of water mist rate supply per unit area of the channel. It can be seen that the relative mass of water mist increases as the water mist rate increases. Conversely, the relative mass of water mist decreases as the temperature difference $\Delta T$ increases. At a low water mist rate $\left(j=20.48 \mathrm{~kg} \mathrm{~m}^{-2}\right.$ $\mathrm{hr}^{-1}$ ), the relative mass of water mist is less than $2 \%$, and the corresponding values of the Nu virtually identical for Nu of airflow as a result of water mist evaporating before reaching to the heated tubes as can be seen from Figure. 5. The main amount of water mist settled on the first row, which justifies the large Nu number values in comparison with the subsequent rows. The last third row almost cooled by the vapor-air flow with a minimum content of water mist as shown in Figure 7 . The ratio of the Nu number measured of tubes exposed to water mist $\left(\mathrm{Nu}_{\mathrm{m}}\right)$ to that when water mist was not suspended $\left(\mathrm{Nu}_{0}\right)$ at the same conditions can be defined as the thermal performance factor. Figure 8 depicts the variation of thermal performance factor with Ra number for a range of water mist rate. 

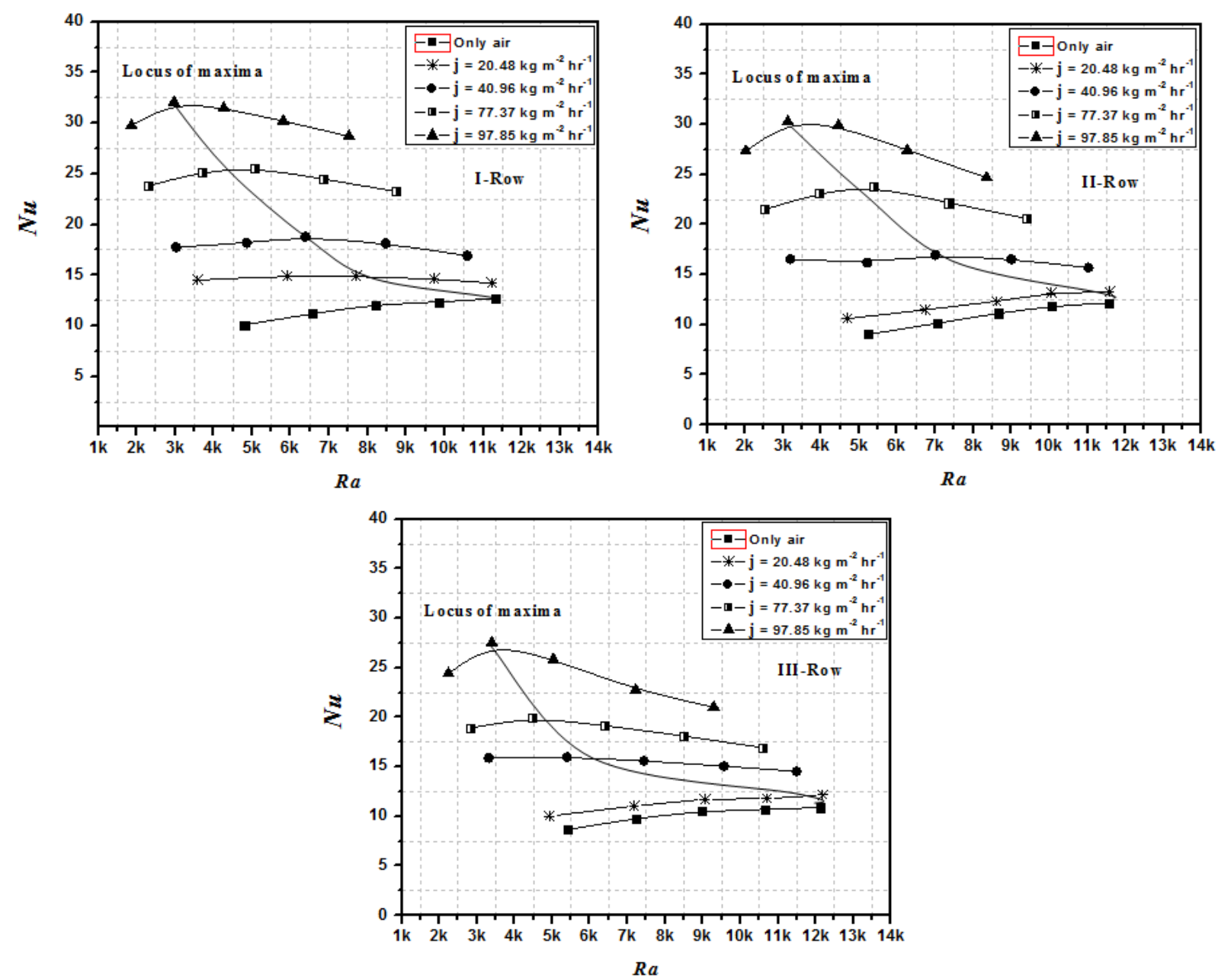

Fig. 5. Variations of Nusselt number with Rayleigh number for various water mist rate

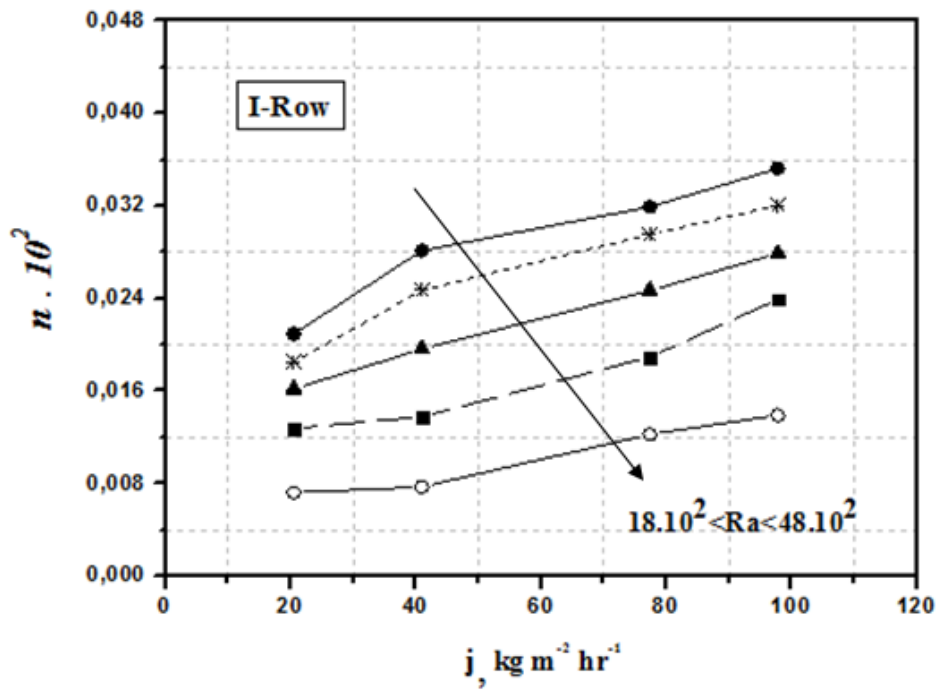

Fig. 6. Variations of the relative mass of water mist settling on the tubes surface with water mist rate 


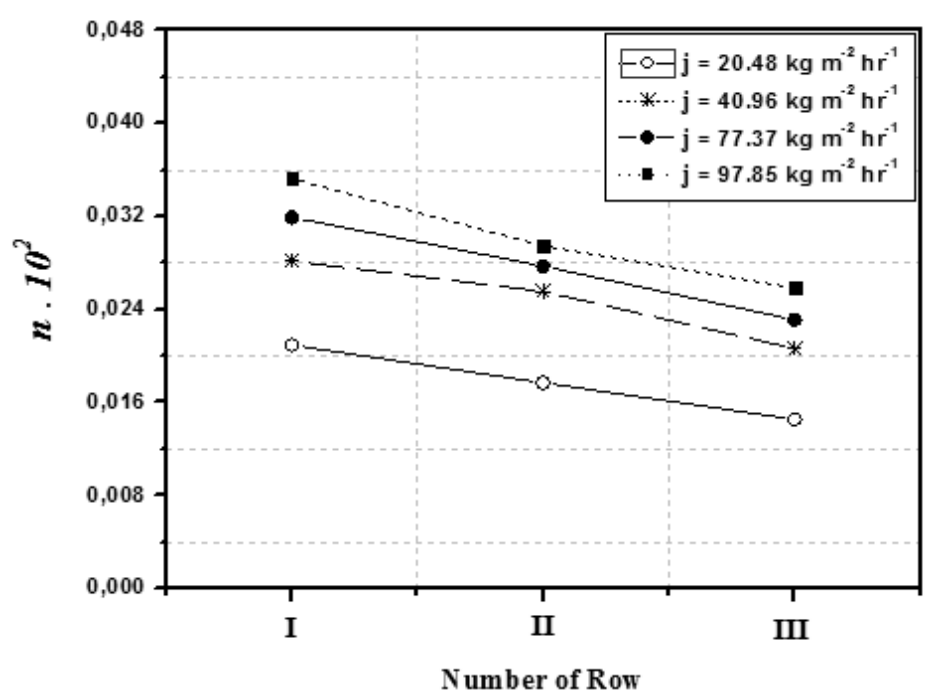

Fig. 7. Variations of the relative mass of water mist settling on the tubes surface as a function of tubes row number
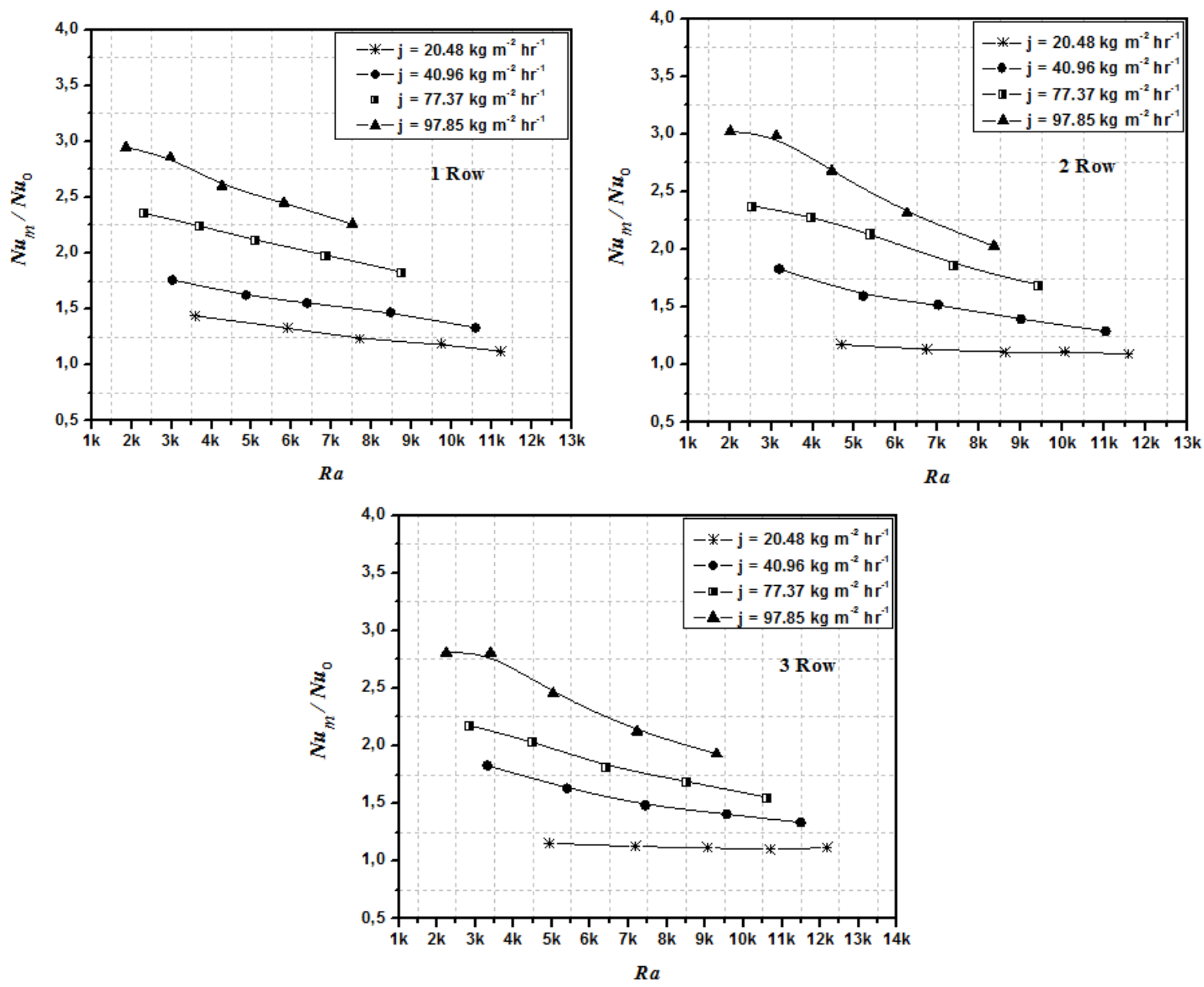

Fig. 8. Variations of thermal performance factor with Ra number for various water mist rate

It is seen that the thermal performance factor tends to increase with increasing water mist rate for all the rows depending on Ra values. The maximum performance factor of about $270 \%$ is achieved for the first row with under $\left(j=97.85 \mathrm{~kg} \mathrm{~m}^{-2} \mathrm{hr}^{-1}\right)$ and is higher than the other water mist rate around 30-120\%. Meanwhile, the maximum performance factor for the second and third rows about $260 \%$ 
and $245 \%$ is achieved with $\left(\mathrm{j}=97.85 \mathrm{~kg} \mathrm{~m}^{-2} \mathrm{hr}^{-1}\right)$. In the present work, the experimental results of the performance factor values obtained by suspended water mist were correlated as functions of dimensionless Rayleigh number ( $\mathrm{Ra}$ ) and Weber number (We) using the least-squares approximation method as given in Eq. (15). Where the coefficient $C=1.95 ; 1.91$ and $1.85 \cdot 10^{7}$ - for the first, second and third tubes bundle. These empirical correlations of performance factor are valid for all range of water mist rates and $\left(\mathrm{Ra}=18 \cdot 10^{2} \sim 12 \cdot 10^{3}\right)$ with absolute deviations between the experimental and predictions data are $11 \%$. as shown in Figure 9.

$\frac{N u_{m}}{N u_{0}}=1+C R a^{-0.79} W e^{0.67}$

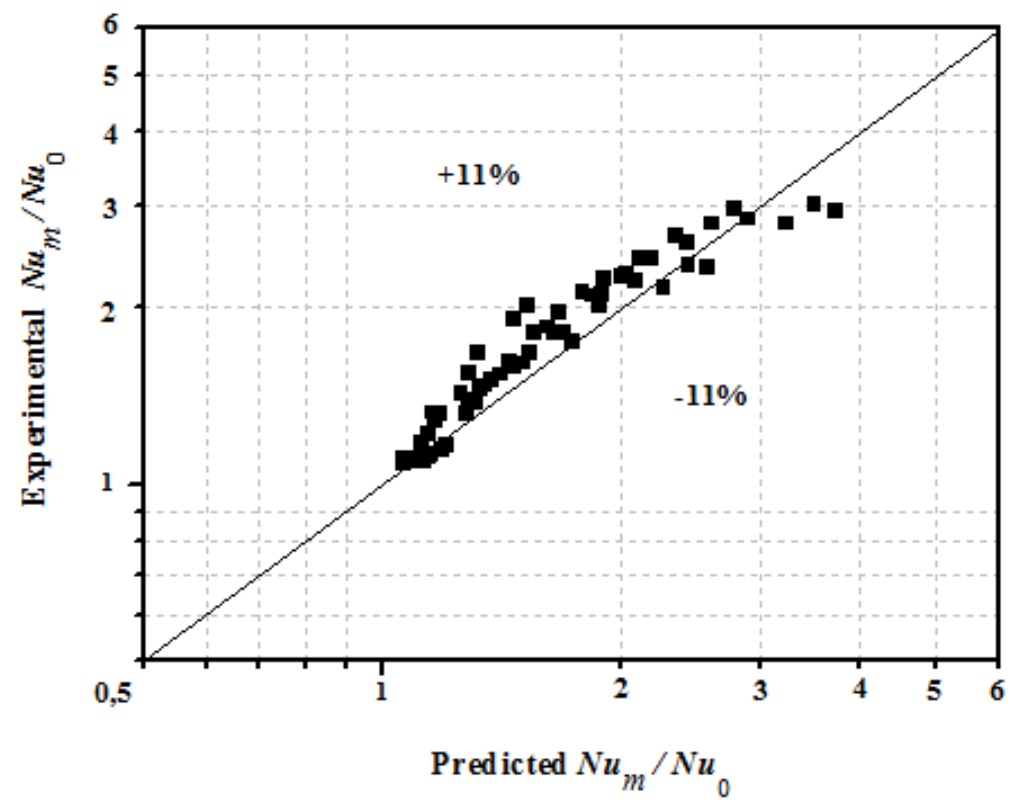

Fig. 9. Comparison between predicted and experimental thermal performance factor

\section{Conclusions}

In this study, the water mist was suspended into the air and their effect on the natural convection heat transfer performance of the tube bundle was investigated experimentally. The attention was focused on the micro-sized and low rates of water mist $\left(j=20.48-97.85 \mathrm{~kg} \mathrm{~m}^{-2} \mathrm{hr}^{-1}\right)$. The main conclusions based on the experimental results can be summarized as follow. The air/water mist cooling is an effective technique to remove a great deal of heat from PHRS and the heat transfer rate could be increased significantly, the Nu number increases by about 148\%, 144\%, and 128\% respectively for all tubes row compared with single-phase airflow under $\left(\mathrm{j}=97.85 \mathrm{~kg} \mathrm{~m}^{-2} \mathrm{hr}^{-1}\right)$. The thermal performance increased significantly by suspending water mist, the present experimental results show up to $270 \%$ of thermal enhancement for all tubes row under a range of water mist rate. The empirical formula is derived, which correlates the performance factor as functions of dimensionless Rayleigh number ( $\mathrm{Ra}$ ) and Weber number (We).

\section{References}

[1] Bezlepkin, Vladimir, Sergey Semashko, Sergey Alekseev, Marina Ivanova, Teimuraz Vardanidze, and Yuriy Petrov. "Improvement of the System for Passive Heat Removal Through Steam Generators (SG PHRS) on NPP With VVER- 
1200 in the Light of "Fukushima" Accident." In 2014 22nd International Conference on Nuclear Engineering. American Society of Mechanical Engineers Digital Collection, 2014.

https://doi.org/10.1115/ICONE22-30240

[2] International Atomic Energy Agency. "Natural Circulation Phenomena and Modelling for Advanced Water Cooled Reactors." IAEA TECDOC-1677 (2012).

[3] Morozov, Andrey, and Alexandra Soshkina. "Passive Core Cooling Systems for Next Generation NPPs: Characteristics and State of the Art." Proceedings of IYNC, Interlaken, Switzerland 1, no 236 (2008): 20-26.

[4] üseyin Ayhan, H., and Cemal Niyazi Sökmen. "Determination of Geometrical and Operating Parameters of PRHRS for VVER Reactors: Cooling by Natural Circulation of Atmospheric Air." In: The $24^{\text {th }}$ International Conference Nuclear Energy for New Europe, Portoroz ', Slovenia, 2015.

[5] Liu, Yanbin, Xuesheng Wang, Xiangyu Meng, and Dawei Wang. "A review on tube external heat transfer for passive residual heat removal heat exchanger in nuclear power plant." Applied Thermal Engineering 149 (2019): 14761491.

https://doi.org/10.1016/j.applthermaleng.2018.11.114

[6] Ayhan, Hüseyin, and Cemal Niyazi Sökmen. "Design and modeling of the passive residual heat removal system for VVERs." Annals of Nuclear Energy 95 (2016): 109-115.

https://doi.org/10.1016/i.anucene.2016.05.003

[7] Ayhan, Hüseyin, and Cemal Niyazi Sökmen. "Investigation of passive residual heat removal system for VVERs: Effects of finned type heat exchanger tubes." Applied Thermal Engineering 108 (2016): 466-474. https://doi.org/10.1016/i.applthermaleng.2016.07.128

[8] Baruah, Monoj, Anupam Dewan, and Pinakeswar Mahanta. "Performance of Elliptical Pin Fin Heat Exchanger with Three Elliptical Perforations." CFD letters 3, no. 2 (2011): 65-73.

[9] Nemati, Hossain, and Mohammad Moghimi. "Numerical study of flow over annular-finned tube heat exchangers by different turbulent models." CFD Letters 6, no. 3 (2014): 101-112.

[10] Skrypnik, A. N., A. V. Shchelchkov, I. A. Popov, D. V. Ryzhkov, S. A. Sverchkov, Yu V. Zhukova, A. D. Chornyi, and N. N. Zubkov. "Thermohydraulic Efficiency of Tubes with Internal Spiral Finning." Journal of Engineering Physics and Thermophysics 91, no. 1 (2018): 52-63. https://doi.org/10.1007/s10891-018-1718-y

[11] Nfawa, Sadeq Rashid, Abd Rahim Abu Talib, Siti Ujila Masuri, Adi Azriff Basri, and Hasril Hasini. "Heat Transfer Enhancement in A Corrugated-Trapezoidal Channel Using Winglet Vortex Generators." CFD Letters 11, no. 10 (2019): 69-80.

[12] Martinez, E., W. Vicente, G. Soto, and M. Salinas. "Comparative analysis of heat transfer and pressure drop in helically segmented finned tube heat exchangers." Applied thermal engineering 30, no. 11-12 (2010): 1470-1476. https://doi.org/10.1016/j.applthermaleng.2010.03.007

[13] Kumari, Niru, Vaibhav Bahadur, Marc Hodes, Todd Salamon, Paul Kolodner, Alan Lyons, and Suresh V. Garimella. "Analysis of evaporating mist flow for enhanced convective heat transfer." International Journal of Heat and Mass Transfer 53, no. 15-16 (2010): 3346-3356.

https://doi.org/10.1016/i.ijheatmasstransfer.2010.02.027

[14] Kim, Kyoung Hoon, Hyung-Jong Ko, Kyoungjin Kim, and Horacio Perez-Blanco. "Analysis of water droplet evaporation in a gas turbine inlet fogging process." Applied Thermal Engineering 33, (2012): 62-69. https://doi.org/10.1016/i.applthermaleng.2011.09.012

[15] Bian, Qingfei, Jin Wang, Yi-tung Chen, Qiuwang Wang, and Min Zeng. "Numerical investigation of mist/air impingement cooling on ribbed blade leading-edge surface." Journal of environmental management 203, (2017): 1062-1071. https://doi.org/10.1016/i.jenvman.2017.05.052

[16] Abed, A. H., V. A. Klimova, S. E. Shcheklein, and V. M. Pakhaluev. "On the possibility to improve heat transfer of a sphere by natural convection and water mist." In Journal of Physics: Conference Series, vol. 1382, no. 1, p. 012124. IOP Publishing, 2019. https://doi.org/10.1088/1742-6596/1382/1/012124

[17] Khangembam, Chunkyraj, Dushyant Singh, Jishnu Handique, and Kuldeep Singh. "Experimental and numerical study of air-water mist jet impingement cooling on a cylinder." International Journal of Heat and Mass Transfer 150 (2020): 119368. https://doi.org/10.1016/j.ijheatmasstransfer.2020.119368

[18] Elwekeel, Fifi NM, and Antar MM Abdala. "Effects of mist and jet cross-section on heat transfer for a confined air jet impinging on a flat plate." International Journal of Thermal Sciences 108, (2016): 174-184. https://doi.org/10.1016/i.ijthermalsci.2016.05.007 
[19] Lang, Robert J. "Ultrasonic atomization of liquids." The journal of the acoustical society of America 34, no. 1 (1962): 6-8.

https://doi.org/10.1121/1.1909020

[20] Abed A. H., Shcheklein, S. E., and V. M. Pakhaluev. "Experimental investigation of hydrodynamics and heat transfer characteristics of Two-Phase Gas/Liquid mist flow in tandem arranged heated spheres." Вестник ЮжноУральского государственного университета. Серия: Энергетика 19, по. 2 (2019). https://doi.org/10.14529/power190202

[21] Moffat, Robert J. "Describing the uncertainties in experimental results." Experimental thermal and fluid science 1, no. 1 (1988): 3-17. https://doi.org/10.1016/0894-1777(88)90043-X

[22] Kline, Stephen J. "Describing uncertainty in single sample experiments." Mech. Engineering 75 (1953): 3-8. 